\title{
Cluster decay of the high-lying excited states in ${ }^{14} \mathbf{C}^{*}$
}

\section{Yanlin Ye $\mathbf{e}^{\dagger}$ Zhengyang Tian, Jing Li}

School of Physics and State Key Laboratory of Nuclear Physics and Technology, Peking

University, Beijing, 100871, China

E-mail: yeyLdpku.edu.cn

\begin{abstract}
Aimed at investigating the molecular structure in high-lying resonant states in ${ }^{14} \mathrm{C}$, a clustertransfer experiment, ${ }^{9} \mathrm{Be}\left({ }^{9} \mathrm{Be},{ }^{14} \mathrm{C} \rightarrow \alpha+{ }^{10} \mathrm{Be}\right) \alpha$ at an incident energy of $45 \mathrm{MeV}$, was carried out. This reaction possesses a very large $Q$-value, which is therefore an excellent case to select the reaction mechanism and the final states in outgoing nuclei. The high-lying resonances in ${ }^{14} \mathrm{C}$ are reconstructed for three sets of well separated final states in ${ }^{10} \mathrm{Be}$. The results confirm the previous decay measurements with improved decay-channel selections and exhibit also a new state at 23.5(1) MeV. The resonant states at 22.4(3) and 24.0(3) MeV decay almost exclusively into the typical molecular states at about $6 \mathrm{MeV}$ in ${ }^{10} \mathrm{Be}$, implying a well developed cluster structure in these high-lying states in ${ }^{14} \mathrm{C}$. Further measurements of more states of this kind are expected.
\end{abstract}

The 26th International Nuclear Physics Conference

11-16 September, 2016

Adelaide, Australia

*This work is supported by the 973 Program of China (No. 2013CB834402) and the NSFC (No. 11535004)

†peaker. 


\section{Introduction}

Clustering is one of the fundamental aspects in structuring the light nuclei[ [प, []], although it appears also quite frequently in heavy nuclei such as the $\alpha$ particle formation and decay[B]]. For isospin-asymmetrical nuclei, the presence of valence nucleons surrounding the cores may help to stabilize these multi-body quantum systems, similar to the molecular configuration for atomic systems[四, 口, 团]. It is well established that neutron-excess beryllium isotopes are good candidates for molecular structure due to its richness of valence neutron combinations built on a well established $\alpha+\alpha$ rotor of ${ }^{8} \mathrm{Be}[[1], \mathbf{6}, \mathbb{Z}, \mathbb{Z}, \mathbf{8}]$. A logical extension to such ideas goes to carbon isotopes, which possess three centers defined by three tightly-bound $\alpha$-particles[四]. Theoretically both triangle and linear-chain configurations were proposed for carbon isotopes, based on the molecular-type potential between two $\alpha$ clusters [四, Q $]$ ].

The famous cluster state, the so called Hoyle state, at an excitation energy of $7.65 \mathrm{MeV}$ in ${ }^{12} \mathrm{C}$, was understood as an $\alpha$-particle Bose-Einstein (BES) condensation where all $\alpha$-particles stay at the relative S-state[四]. It is predicted that the addition of valence neutrons into the $\alpha$-conjugate nuclei may help to stabilize the chain-state[[0]]. Expectations were given to ${ }^{14} \mathrm{C}$ and ${ }^{16} \mathrm{C}$ isotopes based

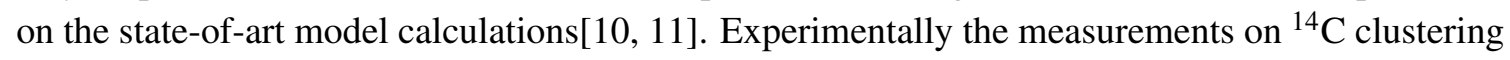
have been proceeded quite intensively during the past decade. In 2003, Soic et al measured the excited states in ${ }^{14} \mathrm{C}$ up to $24 \mathrm{MeV}$ via the cluster transfer reaction, namely ${ }^{7} \mathrm{Li}\left({ }^{9} \mathrm{Be},{ }^{14} \mathrm{C}\right){ }^{2} \mathrm{H}$ [प2]]. The decay of the high-lying excited states in ${ }^{14} \mathrm{C}$ into the ${ }^{10} \mathrm{Be}+\alpha$ final channel were analyzed according to the reaction $Q$-values corresponding to the ground- and first excited-states $\left(3.4 \mathrm{MeV}, 2^{+}\right)$, and the states close to $6 \mathrm{MeV}$, in ${ }^{10} \mathrm{Be}$. The four excited states at around $6 \mathrm{MeV}\left(2^{+}, 1^{-}, 0^{+}, 2^{-}\right)$in

${ }^{10} \mathrm{Be}$ are featured by typical molecular structure built on $2-\alpha$ cores, with both $\sigma$ - and $\pi$-bindings [[13]. Thus based on the structural link (wave-function overlap) between the mother and daughter nuclei, the states at 22.4 and $24.0 \mathrm{MeV}$ in ${ }^{14} \mathrm{C}$ were considered to have a structure change compare to other states [[2], since they decay primarily into the $6 \mathrm{MeV}$ states in ${ }^{10} \mathrm{Be}$. The difficulties in this earlier measurement was the large background below the Q-value peaks, which prohibited the reconstruction of the mother nucleus from a clear identification of the decay process. Since then a number of $2 \mathrm{n}$ transfer or $2 \mathrm{p}$ removal experiments were also performed to populate the high-lying excited states in ${ }^{14} \mathrm{C}$. These reactions are more sensitive to the single-particle configurations with oblate or prolate deformations [14, [15, [6, [1]]. The latest resonant $\alpha$-scattering experiments are dedicated to search for cluster states in ${ }^{14} \mathrm{C}$. Based on the observed resonant states some possible molecular-rotation bands or linear-chain states were proposed [18, [9]. However due to the limited energy resolution of this thick-target method, the decays to $6 \mathrm{MeV}$ states in ${ }^{10} \mathrm{Be}$ were not distinguished.

We report here on a reaction ${ }^{9} \mathrm{Be}\left({ }^{9} \mathrm{Be},{ }^{14} \mathrm{C}\right){ }^{4} \mathrm{He}$ to study the cluster states in ${ }^{14} \mathrm{C}$. This reaction channel has an extremely large $Q$-value compared to other competing reaction channels. The highlying states in ${ }^{14} \mathrm{C}$ are reconstructed from the ${ }^{10} \mathrm{Be}+\alpha$ decay channels. Three sets of final states in the daughter nucleus ${ }^{10} \mathrm{Be}$, namely the ground-, $2^{+}$and $\sim 6 \mathrm{MeV}$ states, are identified and used to track the possible decay paths. 


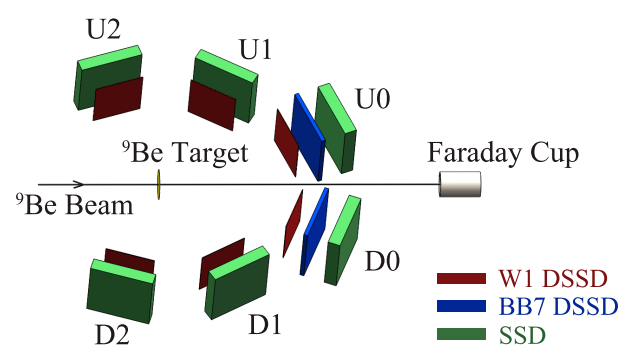

Figure 1: A schematic view of the experimental setup.

\section{Experiment}

A detailed description of the experimental setup can be found in Ref.[20]. The experiment was performed at the HI-13 tandem accelerator at China Institute of Atomic Energy (CIAE). A ${ }^{9} \mathrm{Be}$ beam at $45 \mathrm{MeV}$ and with an intensity of about 7 enA was incident on a $0.9 \mu \mathrm{m}$ thick selfsupporting ${ }^{9} \mathrm{Be}$ target. Fig. $\mathrm{W}$ gives a schematic drawing of the detection system. Reaction products were detected by six particle telescopes, namely U0, U1, U2, D0, D1, D2. The front faces of the forward telescopes (U0 and D0) were positioned at $140 \mathrm{~mm}$ from the target and centered at \pm 23 ąã relative to the beam axis. Each of them consisted of one layer of double-sided silicon strip detector (DSSD) with a thickness of $60 \mu \mathrm{m}$ (W1-60), one DSSD with a thickness of $500 \mu \mathrm{m}$ (BB7-500) and one layer of large-size silicon detector (SSD) with a thickness of $1500 \mu \mathrm{m}$ (MSX40-1500). The sensitive area is $50 \mathrm{~mm} \times 50 \mathrm{~mm}$ for W1-60, and $64 \mathrm{~mm} \times 64 \mathrm{~mm}$ for BB7-500 and MSX40-1500. The front and back faces are both divided into 16 strips for W1-60, and 32 strips for BB7-500. Each of the other four telescopes consisted of one W1-60 and one MSX40-1500. U1 and D1 were located at a distance of $116 \mathrm{~mm}$ from the target, and centered at large angles of $\pm 60^{\circ}$, while U2 and D2 at $114 \mathrm{~mm}$ and $\pm 109^{\circ}$. The forward telescopes (U0 and D0) were primarily used to detect the decay fragments from the excited ${ }^{14} \mathrm{C}$. Fig. $\square$ demonstrates an example (U0) of the particle identification performance of the telescope, which is good enough to discriminate the isotopes to be used in the present study. Other telescopes at larger angles were employed to detect the recoiled $\alpha$-particles. Energy calibration of the detectors was realized using the $\alpha$ sources [ [2]]. The energy match for all silicon strips in one detector was achieved according to the uniform calibration method as described in [2].

\section{Analysis and results}

Events with ${ }^{10} \mathrm{Be}+\alpha$ two-fold coincidence, taken from the two opposite forward telescopes (U0 and D0), were selected for the present analysis. The remaining undetected nucleus is expected to be an $\alpha$-particle, of which the momentum and energy can be deduced according to the respective conservation requirements. The reaction $Q$-value can be deduced using the definition:

$$
\begin{aligned}
Q & =E_{\text {tot }}-E_{\text {beam }} \\
& =E_{10 \mathrm{Be}}+E_{\alpha 1}+E_{\alpha 2}-E_{\text {beam }}
\end{aligned}
$$




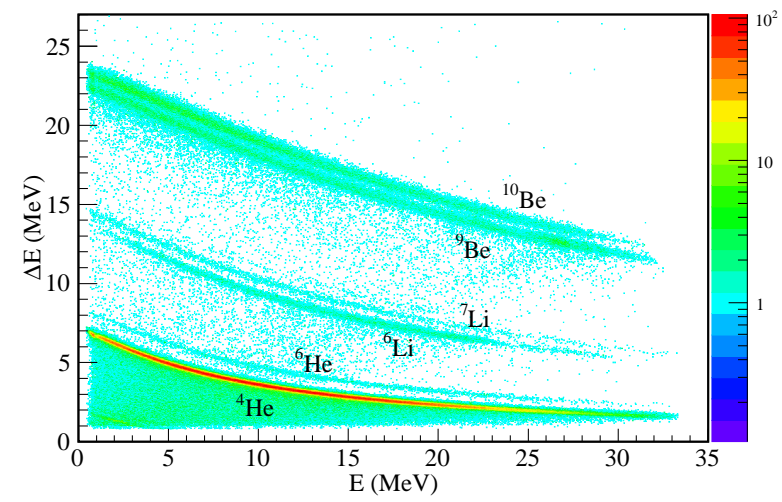

Figure 2: Particle identification performance for the U0 telescope, with $\triangle E$ the energy loss in the W1-60 detector and $E$ the remaining energy in the BB7-500 detector.

As displayed in Fig. [1, the $Q$-value for final particles all at their ground states, $Q_{\mathrm{ggg}}$, is -1.91 $\mathrm{MeV}$ for the previous measurement [ㅁ]](Fig. B](a)) and 5.24 MeV for the present work (Fig. B](b)). An extremely large $Q$-value, due to two deeply-bound $\alpha$-particles in the final channel, is characteristic in the present experiment. This is important for a clean selection of the reaction mechanism. In the figure, the peak next to $Q_{g g g}$ corresponds to the first excited state $\left(3.4 \mathrm{MeV}, 2^{+}\right)$, whereas the further next one at about $6 \mathrm{MeV}$ excitation includes the four adjacent states, in ${ }^{10} \mathrm{Be}$ [[2]]. Since other possible reaction channels, together with corresponding angular range, lead to much lower $Q$-values, the peaks in Fig. B(b) have almost no contaminations. The broad background spectrum below the peaks is much less significant in the present case (Fig. B B(b)) than in the previous work (Fig. B](a)). We note that this background can further be reduced when applying more strick timing window to rule out the event mixing. This timing analysis is still underway. The shape of the present $Q$-value spectrum was verified by using the completely detected 3-fold coincident events. It is evident that the present measurement provides the possibility to unambiguously identify the resonant states in ${ }^{14} \mathrm{C}$ according to its decay to each final state in ${ }^{10} \mathrm{Be}$.

Gated on the three $Q$-value peaks shown in Fig. B](b) and using the method described in Ref.[[, [23], the high-lying resonant states in ${ }^{14} \mathrm{C}$ can be reconstructed from the ${ }^{14} \mathrm{C} \rightarrow \alpha+{ }^{10} \mathrm{Be}$ decay channel, and the results are presented in Fig. $Q$ G(b), together with previous results (Fig. QG(a)) for comparison. The results are plotted as empty diamonds, filled circles and empty triangles for decaying to the ground, first excited and $\sim 6 \mathrm{MeV}$ states in ${ }^{10} \mathrm{Be}$, respectively. For all three cases our detection acceptances (efficiencies) are in favor of the higher energy sides, owing to the angular coverage of the detector setups.

It can be seen from Fig. $\boxplus$ that most states coincide very well with each other for the two measurements, demonstrating the reliability of the present experimental and data analysis methods. In case of decaying into ${ }^{10} \mathrm{Be}_{\mathrm{gs}}$ (empty diamonds), the previously observed strong peak at $16.4 \mathrm{MeV}$ is well confirmed in our experiment, while the peaks at $19.8 \mathrm{MeV}$ and $20.6 \mathrm{MeV}$ are much more significant in the present measurement. As for ${ }^{10} \mathrm{Be}\left(3.4 \mathrm{MeV}, 2^{+}\right)$final state (filled circles), in addition to the confirmation of states at 19.8 and $21.4 \mathrm{MeV}$, we have clearly observed a new peak at 23.5(1) $\mathrm{MeV}$, as indicated by the arrow. This peak appears very weakly in the previous measurement due to 


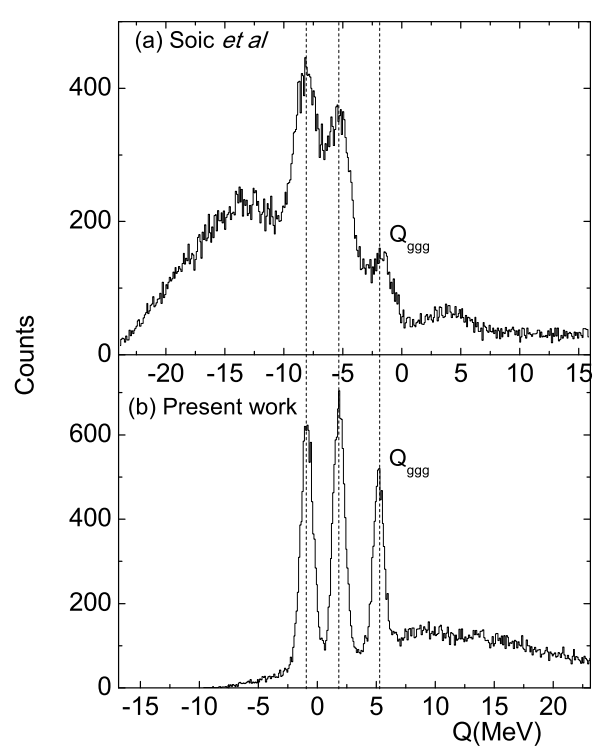

Figure 3: $Q$-value (reaction energy) spectra for (a) the reaction ${ }^{7} \mathrm{Li}\left({ }^{9} \mathrm{Be}, \alpha{ }^{10} \mathrm{Be}\right)^{2} \mathrm{H}[\mathrm{L2}]$ and (b) the present measurement of ${ }^{9} \mathrm{Be}\left({ }^{9} \mathrm{Be}, \alpha^{10} \mathrm{Be}\right) \alpha$. The spectra are aligned according to the corresponding peaks. The vertical dashed lines are plotted to guide the eyes.

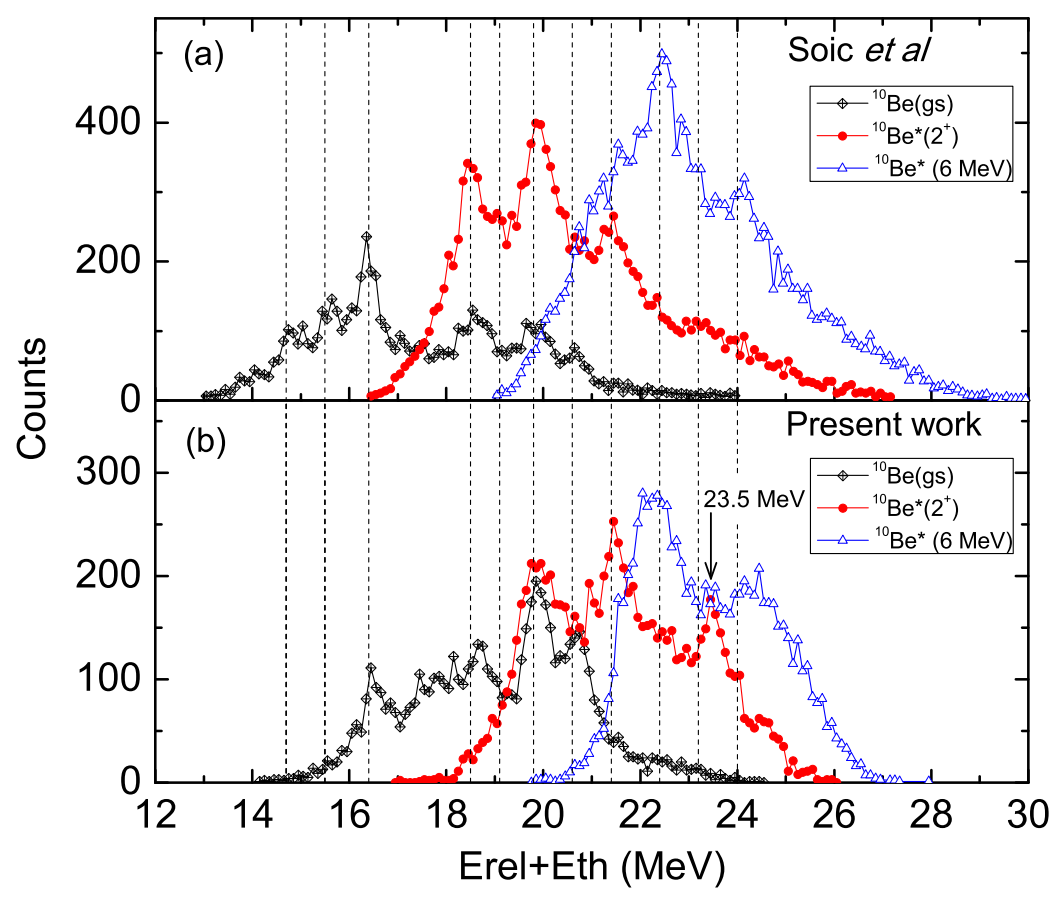

Figure 4: The excited states in ${ }^{14} \mathrm{C}$ reconstructed from the $\alpha+{ }^{10} \mathrm{Be}$ cluster-decay channel, with the upper panel (a) taken from the previous measurement[[1] ] and the lower panel (b) from the present work. The decay final states are selected for the ground, first excited and $\sim 6 \mathrm{MeV}$ states in ${ }^{10} \mathrm{Be}$. 
the low acceptance there. The uncertainty of the peak positions for the present experiment is about $80 \mathrm{keV}$, dominated by the systematic errors in the energy calibration of the telescopes (about 50 $\mathrm{keV}$ in the interested energy range) and in the estimation of the dead-layer thickness of the silicon detector. Again we see a good match of the ${ }^{14} \mathrm{C}$-states which decay simultaneously into ${ }^{10} \mathrm{Be}_{\mathrm{gs}}$ and ${ }^{10} \mathrm{Be}\left(3.4 \mathrm{MeV}, 2^{+}\right)$. Most of these resonant states have also been observed in recent resonant scattering experiment [18, [9], where spin assignment were made using the angular correlation method. The states at very high energy of 23.5(1) $\mathrm{MeV}$ has only been identified in the present experiment, which might be an extension of the tentatively proposed molecular rotation band (see Fig.12 in Ref.[ए8]]).

The states at 22.4(3) and 24.0(3) MeV (empty triangles in the Fig. [1]) decay almost exclusively into the $\sim 6 \mathrm{MeV}$ states in ${ }^{10} \mathrm{Be}$. implying a possible change of structure configuration for these very high-lying states. It should be noted that the uncertainty of the peak positions here is larger due to the energy deviation of the four states around $6 \mathrm{MeV}$ in ${ }^{10} \mathrm{Be}$. It is widely accepted that these $\sim 6$ $\mathrm{MeV}$ states in ${ }^{10} \mathrm{Be}$ are band heads of reflection-asymmetric molecular-rotation [[13]]. Considering the structural-link in the decay, it is reasonable to consider these very high-lying resonances in ${ }^{14} \mathrm{C}$ as well developed three-center molecular states. We confirmed two states of this kind here with much clearer $Q$-value selection as demonstrated in Fig. [3. It is expected that more states of this kind might be observed at higher energy range if one extend the measurement to larger opening angle for the decaying fragment pair. It is also important to determine the spins of these states based on the experimental information.

\section{Conclusion}

We have applied the reaction ${ }^{9} \mathrm{Be}\left({ }^{9} \mathrm{Be}, \alpha{ }^{10} \mathrm{Be}\right) \alpha$ to populate and reconstruct the high-lying resonant states in ${ }^{14} \mathrm{C}$. This reaction is of extremely large $Q$-value, making it an excellent case to select the reaction mechanism and the final states in the outgoing daughter nucleus. The background contamination was largely reduced compared to previous measurements, owing to the selected reaction channel, and the excellent energy and position resolutions of the silicon-strip detectors. The high-lying excited states in ${ }^{14} \mathrm{C}$ are reconstructed with respect to three sets of well discriminated final states in ${ }^{10} \mathrm{Be}$. Most of the presently observed states, which decay to ${ }^{10} \mathrm{Be}_{\mathrm{gs}}$ and to ${ }^{10} \mathrm{Be}\left(3.4 \mathrm{MeV}, 2^{+}\right)$, agree well with the previous observations [12]]. A new state at $23.5 \mathrm{MeV}$ is clearly identified in the present work. The states at 22.4 and $24.0 \mathrm{MeV}$ in ${ }^{14} \mathrm{C}$ decay dominantly into the $\sim 6 \mathrm{MeV}$ states in ${ }^{10} \mathrm{Be}$. The latter possess typical molecular structure, implying a well developed three-center molecular configuration in the mother states in ${ }^{14} \mathrm{C}$. The present results, based on the significantly improved selection of reaction and decay paths, support the richness of cluster formation and decay in excited states of light neutron-rich nuclei. More measurements of this kind, especially with the determination of the decay-path and the spin, are certainly encouraged.

\section{References}

[1] W. von Oertzen, M. Freer and Y. Kanada-Enyo, Phys. Rep., 432: 43-113 (2006)

[2] Y.L. Ye, L.H. Lv, Z.X. Cao, J. Xiao, Chin. Phys. C, 36: 127-131 (2012)

[3] Y.B. Qian and Z.Z. Ren, Phys. Rev. C, 88: 044329 (2013) 
[4] H. Horiuchi, K. Ikeda and K. Kato, Prog. Theor. Phys. Suppl., 192: 1-238 (2013)

[5] Z.H. Yang, Y.L. Ye, Z.H. Li et al, Phys. Rev. Lett., 112: 162501 (2014)

[6] Z.H. Yang, Y.L. Ye, Z.H. Li et al, Sci. China-Phys. Mech. Astron. 57: 1613-1617 (2014)

[7] Z.H. Yang, Y.L. Ye, Z.H. Li et al, Phys. Rev. C, 91: 024304 (2015)

[8] M.Lyu, Z.Z. Ren, B. Zhou, Y. Funaki, H. Horiuchi, P. Ropke, G. Schuck, A. Tohsaki, C. Xu, and T. Yamada, Phys. Rev. C, 93: 054308 (2016)

[9] D.H. Wikinson, Nucl. Phys. A, 452: 296 (1986)

[10] N. Itagaki, S. Okabe, K. Ikeda and I. Tanihata, Phys. Rev. C, 64: 014301 (2001)

[11] T. Suhara and Y. Kanada-Enyo, Phys. Rev. C, 82: 044301 (2010)

[12] N. Soic, M. Freer, L. Donadille et al, Phys. Rev. C, 68: 014321 (2003)

[13] W. von Oertzen, Z. Phys. Rev. A, 357: 355-365 (1997)

[14] M. Milin, S. Cherubini, T. Davinson et al, Nucl. Phys. A, 730: 285-298 (2004)

[15] W. von Oertzen, H.G. Bohlen, M. Milin et al, Eur. Phys. J. A, 21: 193-215 (2004)

[16] D.L. Price, M. Freer, N.I. Ashwood et al, Phys. Rev. C, 75: 014305 (2007)

[17] P.J. Haigh, N.I. Ashwood, T. Bloxham et al, Phys. Rev. C, 78: 014319 (2008)

[18] M. Freer, J.D.Malcolm, N.L.Achouri et al, Phys. Rev. C, 90: 054324 (2014)

[19] A. Fritsch, S. Beceiro-Novo, D.Suzuki et al, Phys. Rev. C, 93: 014321 (2016)

[20] Z. Y. Tian, Y. L. Ye, Z. H. Li, C. J. Lin, et al., Chinese Physics C, 40: 111001 (2016)

[21] J. Chen, J.L. Lou, Y.L. Ye et al, Phys. Rev. C, 93: 034623(2016)

[22] R. Qiao, Y.L. Ye, J. Wang et al, IEEE Tran. Nucl. Sci., 61: 596-601 (2014)

[23] L.H. Lv, Y.L. Ye, D.X. Jiang et al, Chin. Phys. C, 35: 891—895 (2011) 